\title{
Noninvasive evaluation of craniovertebral junction instability in 2 patients following Chiari decompression with rigid C-collar immobilization: illustrative cases
}

\author{
Kerrin S. Sunshine, BA, ${ }^{3}$ Theresa A. Elder, MD, ${ }^{1,3}$ and Krystal L. Tomei, MD, MPH ${ }^{1-3}$ \\ ${ }^{1}$ Department of Neurological Surgery, University Hospitals Cleveland Medical Center, Cleveland, Ohio; '2Division of Pediatric Neurosurgery, University Hospitals Rainbow \\ Babies \& Children's Hospital, Cleveland, Ohio; and ${ }^{3}$ Case Western Reserve University School of Medicine, Cleveland, Ohio
}

\begin{abstract}
BACKGROUND The complex Chiari malformation has been identified in a subset of Chiari patients at higher risk for worsening symptoms following Chiari decompression. Although parameters such as the clivoaxial angle and the perpendicular distance of the dens to the line from the basion to the inferoposterior part of the $\mathrm{C} 2$ body $(\mathrm{pBC} 2$ ) have been evaluated to help with the prediction of risk, the decision to pursue an occipitocervical fusion in lower-risk patients does not come without inherent risk.

OBSERVATIONS The authors present 2 patients who had symptoms of worsening ventral brainstem compression following Chiari decompression, neither of whom was categorized in the highest risk category for occipitocervical instability. In addition, neither patient had gross instability on radiographic imaging. A trial with rigid C-collar immobilization provided relief of symptoms in both patients and allowed reassurance of the likelihood of success of occipitocervical fusion.
\end{abstract}

LESSONS In patients without clear radiographic instability following Chiari decompression, a C-collar trial may provide a noninvasive option for assessing the potential success of occipitocervical fusion.

https://thejns.org/doi/abs/10.3171/CASE20114

KEYWORDS complex Chiari malformation; suboccipital decompression; atlantoaxial instability

The Chiari I malformation (CIM) is a congenital anomaly often treated with suboccipital decompression surgery. Over time, within large series of patients with Chiari malformations, a small subset of patients with CIM was found to worsen following decompression, presenting with ventral compression and signs and symptoms of bulbar dysfunction with a reported incidence of $4 \%-31 \% .{ }^{1-4}$ Further evaluation of this subset of patients with an increased symptom burden led to an improved classification of these patients, including a novel category of Chiari 1.5 malformation. "Chiari 1.5" was defined as CIM with additional radiographic evidence of brainstem and obex herniation below the level of the foramen magnum. ${ }^{5}$

A subset of patients requiring more frequent surgical intervention than their peers, including occipitocervical fusion following Chiari decompression, was then identified. Thus, the notion of the complex Chiari malformation was added to the spectrum of CIM and Chiari 1.5 to define morphological features that were found to confer a higher likelihood of requiring further surgical intervention. This category was defined as "cerebellar tonsil herniation combined with one or more of the following radiographic findings: brainstem herniation through the foramen magnum (Chiari 1.5 malformation), medullary kink, retroflexed odontoid, abnormal clivoaxial angle, occipitalization of the atlas, basilar invagination, syringomyelia or scoliosis."6

In the treatment of these symptomatic patients, there is controversy regarding to what extent the brainstem compression should be surgically treated, if at all. ${ }^{1,7}$ In current practice, radiographic markers such as the perpendicular distance of the posterior dens to the line from the basion to the inferoposterior part of the $\mathrm{C} 2$ body (pBC2 line), the clivoaxial angle (CXA), and McRae's line are used to help stratify patients at higher risk for needing further intervention after decompression, in addition to flexion-extension radiographs. ${ }^{7}$ However, not all patients with worsening symptoms after decompression possess these radiographic markers.

We present the cases of 2 patients with complex Chiari malformation who underwent decompression and subsequently developed

ABBREVIATIONS $\mathrm{BDI}=$ basion-dens interval; $\mathrm{CIM}=$ Chiari I malformation; $\mathrm{CXA}=$ clivoaxial angle $; \mathrm{MRI}=$ magnetic resonance imaging; $\mathrm{pBC2}=$ perpendicular distance of dens to the line from the basion to the inferoposterior part of the $\mathrm{C} 2$ body.

INCLUDE WHEN CITING Published February 8, 2021; DOI: 10.3171/CASE20114.

SUBMITTED November 13, 2020. ACCEPTED December $9,2020$.

(c) 2021 The authors, CC BY-NC-ND 4.0 (http://creativecommons.org/licenses/by-nc-nd/4.0/). 
symptoms of ventral brainstem compression. Both patients did not possess radiographic markers of high risk for ventral brainstem symptoms and did not have convincing signs of gross craniocervical instability on flexion-extension imaging. Both patients were trialed in a C-collar, resulting in resolution of their symptoms and justifying the need to perform an occipitocervical fusion.

\section{Illustrative Cases}

\section{Case 1}

The patient initially presented at age 13 years after a new-onset syncopal event. She underwent computed tomography followed by magnetic resonance imaging (MRI), which confirmed a Chiari 1.5 malformation. Her history was notable for both occipital and frontal headaches twice per week, without clear exacerbating factors, as well as frequent lightheadedness upon arising and stumbling while walking. She denied any dysphagia and numbness or tingling of the extremities. She was neurologically intact on examination.

The patient had no evidence of syrinx or tethered cord on MRI. However, she was noted to have retroflexion of the odontoid with a CXA of $120^{\circ}$ and a pBC2 line measuring $7.3 \mathrm{~mm}$ (Fig. 1). Radiographs of her cervical spine did not demonstrate any dynamic instability. She was initially managed conservatively for 3 months, at which point her headaches worsened, and she developed frequent palpitations. At this time, the family opted to proceed with Chiari decompression. She underwent a suboccipital craniectomy and $\mathrm{C} 1$ laminectomy with intradural exploration, tonsillar cauterization, and release of a fourth ventricular outflow arachnoid web with expansile duraplasty using DuraMatrix graft material (Stryker). The patient's postoperative course was complicated by nausea and gagging episodes, treated initially as aseptic meningitis with dexamethasone.

At her 1-month follow-up, the patient reported resolution of her headaches with persistent nausea and dry heaving without emesis. She also noted improvement in palpitations and "shakiness" but persistent problems with balance and coordination. She completed a total of 3 steroid courses of increasing taper duration, and at approximately 2.5 months postoperatively, her gagging episodes began improving. Steroids were tapered, and she transitioned to prochlorperazine and subsequently promethazine for the gagging episodes. By 3 months
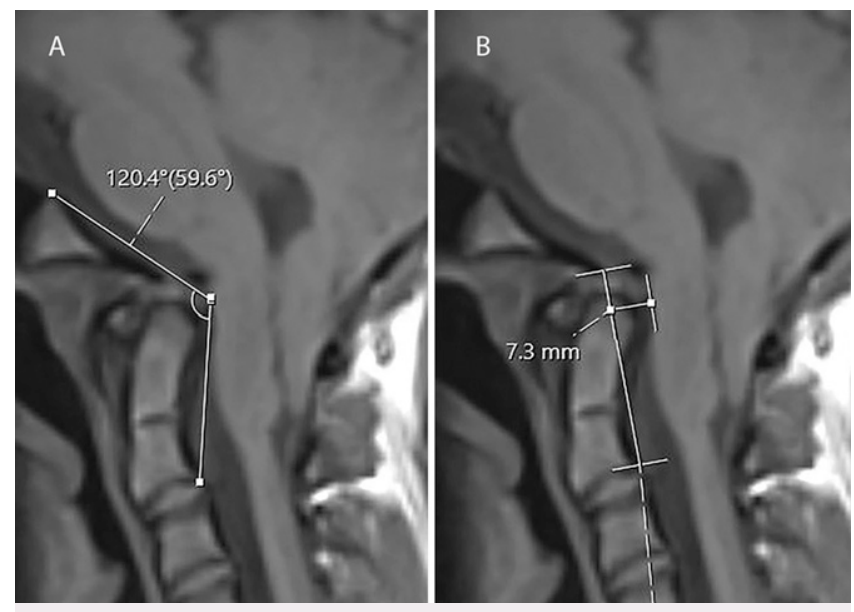

FIG. 1. Measurements prior to decompression. A: CXA measuring $120^{\circ}$. B: pBC2 line measuring $7.3 \mathrm{~mm}$. postoperatively, the episodes had improved but still occurred daily. The result of cerebrospinal fluid analysis was negative for infection, and MRI in neutral and flexion views demonstrated a minimal 1-mm change in her basion-dens interval (BDI) with flexion (Fig. 2). Dynamic C-spine radiographs demonstrated no evidence of gross atlantoaxial instability.

Approximately 6 months postoperatively, despite resolution of her headaches, her episodes of lightheadedness returned, and she continued to have persistent gagging. Given the signs of ventral brainstem compression, without clear radiographic evidence of instability as well as the CXA and $\mathrm{pBC} 2$ measurements, which would have placed her at approximately a $13 \%$ risk of needing an occipitocervical fusion, she was placed in a rigid Miami $\mathrm{J}$ collar to determine whether decreasing neck motion would improve her symptoms. ${ }^{7,8}$ After 1 month in a cervical collar, her gagging and vasovagal symptoms had completely resolved. To rule out any masking of symptoms, she was weaned from the promethazine, and her gagging remained absent. The C-collar was discontinued, after which her symptoms returned along with new neck pain. She subsequently underwent an occiput-to-C2 fusion with an autologous rib graft with the use of a U-bar construct with wiring for fixation (Fig. 3). She was maintained in a halo orthosis for 3 months postoperatively. She had complete resolution of her symptoms following her occipitocervical fusion, and 4 years postoperatively, she was without complications.

\section{Case 2}

The 14-year-old patient had a history of chronic headaches that began after a motor vehicle collision approximately 4 years earlier. She presented for routine follow-up and was noted to have scoliosis and, upon further workup, was found to have a Chiari 1.5 malformation with associated cervical syrinx. She underwent a Chiari decompression with duraplasty at an outside hospital. Approximately 4 months following her surgery, she developed intractable hiccups, left-sided neck pain, and tachycardia. She was managed medically with numerous agents without improvement in her symptoms. She presented to our institution for a seizure workup to evaluate her hiccup etiology. Upon initial imaging, she was noted to have retroflexion of her odontoid with a CXA of $143^{\circ}$ and a pBC2 line measuring $6.6 \mathrm{~mm}$ (Fig. 4). She
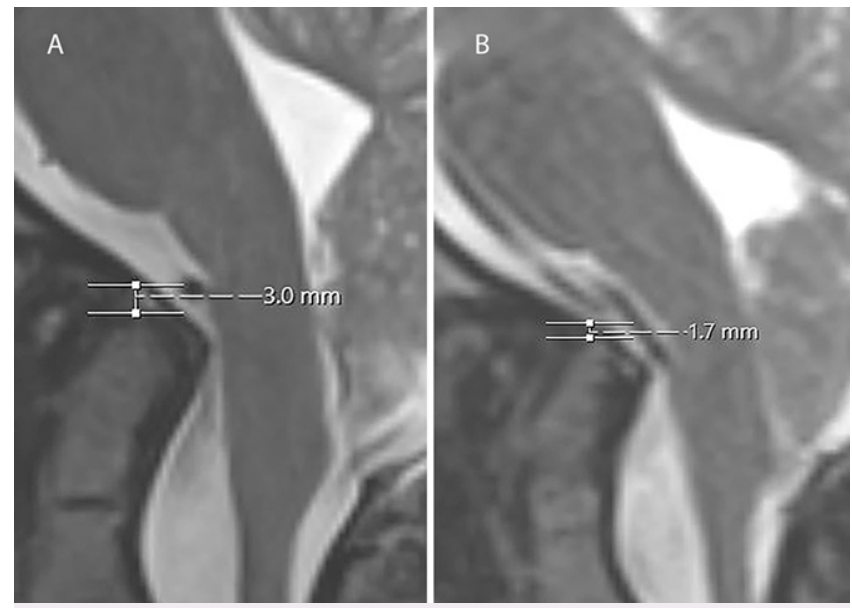

FIG. 2. Dynamic MRI with BDI measurements in flexion (A) and neutral (B) positions. 


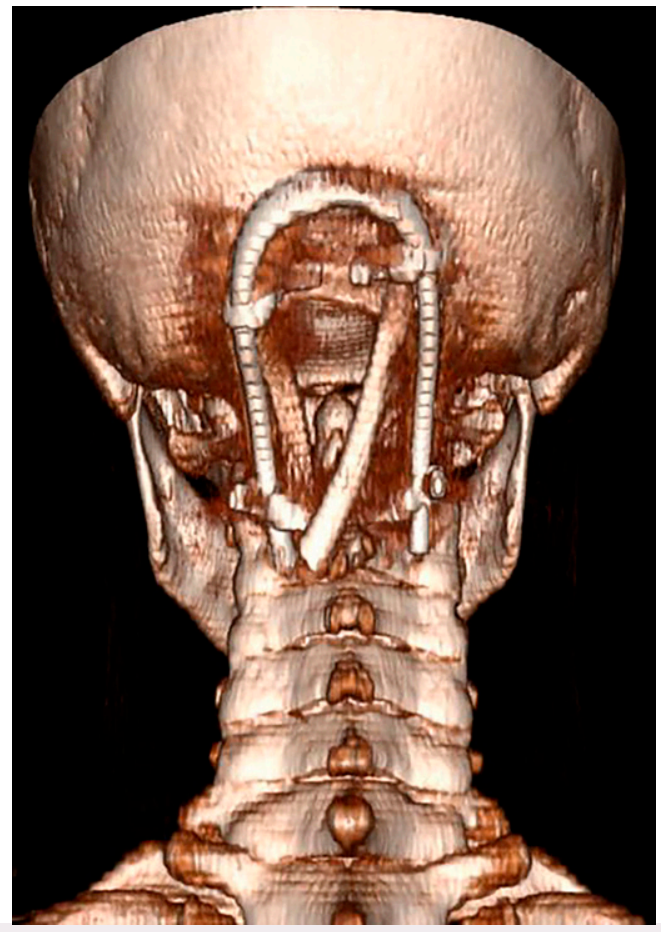

FIG. 3. Fusion construct compensating for the skull defect by using a U-bar rod and laminar and occipital wiring with autologous rib grafts.

underwent flexion-extension radiographic imaging that did not demonstrate significant dynamic instability. She then underwent dynamic MRI of her cervical spine, which demonstrated a neutral BDI of $2.7 \mathrm{~mm}$, a flexion BDI of $2.4 \mathrm{~mm}$, and an extension BDI of $4.1 \mathrm{~mm}$ (Fig. 5). She was evaluated by an otolaryngologist for evidence of oral trauma that could explain a vagus nerve injury. Ultimately, with an unremarkable workup, she was placed in a rigid Miami $\mathrm{J}$ collar, and within a few weeks, her hiccups resolved. She then underwent an occiput-to-C2 fusion with postoperative halo orthosis for 3 months. Her postoperative course was complicated only by a few episodes of halo pin loosening and selflimited meralgia paresthetica resulting from prone positioning, which resolved. Halo removal was performed at 3 months following surgery with complete resolution of her symptoms. Four years postoperatively, she had no further complications, resolution of her syrinx, and no progression of her scoliosis.

\section{Discussion}

\section{Observations}

Reports of ventral brainstem compression symptoms, including vertigo, diplopia, dysphagia, apnea, and other lower cranial nerve signs, following Chiari decompression are limited, with the majority of available literature describing the persistence of symptoms that were initially present preoperatively but nonetheless thought to increase the likelihood of requiring fusion. 6,9 Traditionally, defining ventral brainstem compression in pediatric patients with Chiari was limited to requiring the presence of basilar invagination. ${ }^{10}$ However, it is now known that some patients may have symptomatic ventral compression in the absence of basilar invagination, suggesting craniocervical instability. ${ }^{10}$ These

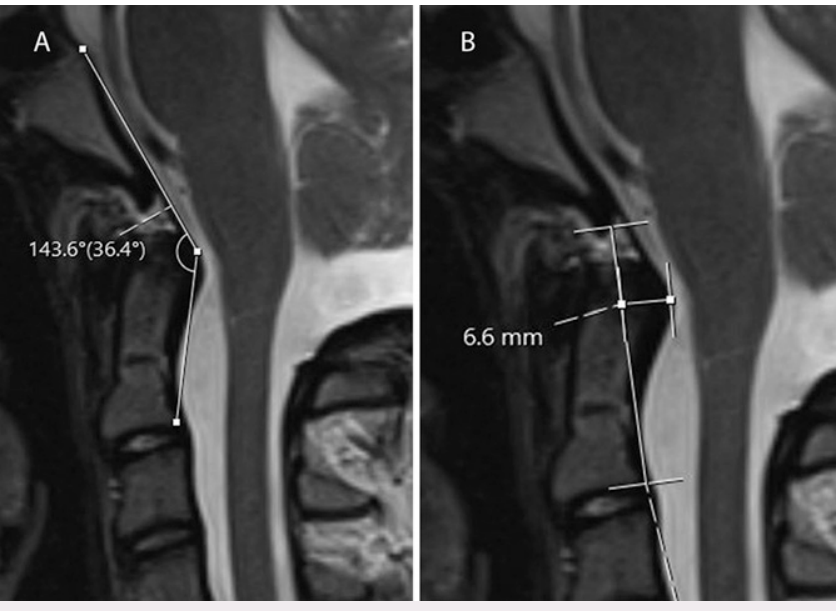

FIG. 4. Measurements following decompression at an outside hospital. A: CXA measuring $143^{\circ}\left(36.4^{\circ}\right.$ acute measurement). B: pBC2 line measuring $6.6 \mathrm{~mm}$.

patients require subsequent occipitocervical fusion, reported to occur in as many as $10 \%$ of patients. ${ }^{10,11}$

Various radiographic markers have been studied to further define instability causing ventral brainstem compression and to stratify risk in patients with occipitocervical instability needing occipitocervical fusion. Grabb and colleagues defined a measurement of odontoid retroflexion: the $\mathrm{pBC} 2$ line, a line drawn from the inferior portion of the clivus to the posterior-inferior aspect of the $\mathrm{C} 2$ vertebral body. ${ }^{3}$ This line shows the maximum perpendicular distance from the base of the inferoposterior point of $\mathrm{C} 2$. Grabb and colleagues found a pBC2 distance $>9 \mathrm{~mm}$ to be associated with symptomatic ventral brainstem compression and the risk of occipitocervical instability. ${ }^{3}$ In 1994, Smoker defined craniocervical angulation by using the CXA. ${ }^{12}$ The CXA represents the angle between the lines drawn from the inferior two-thirds of the clivus and a line drawn from the posterior-inferior $\mathrm{C} 2$ body to the superior posterior aspect of the odontoid. In a large retrospective review, Bollo and colleagues found Chiari 1.5 , a clival-cervical angle $<125^{\circ}$, and basilar invagination each independently placing a patient at higher risk than those with more traditional CIMs of requiring occipitocervical fusion. ${ }^{6}$ Brockmeyer and Spader created a treatment algorithm based on a $\mathrm{pBC} 2$ line cutoff of $9 \mathrm{~mm}$, a CXA cutoff of $125^{\circ}$, and the absence or presence of bulbar and myelopathic symptoms to predict the need for occipitocervical fusion. ${ }^{7}$ A patient with a $\mathrm{pBC} 2$ line $>9 \mathrm{~mm}$ and a $\mathrm{CXA}<125^{\circ}$ was found to most likely need an occipitocervical fusion following Chiari decompression. Conversely, patients with a normal $\mathrm{pBC} 2$ and $\mathrm{CXA}$ did not require occipitocervical fusion following Chiari decompression. In addition, they did not possess clear findings of gross instability on flexion-extension radiographs indicative of joint laxity and occipitocervical instability. The BDI can also help to determine abnormal laxity, with a BDI of $12 \mathrm{~mm}$ or more considered to be pathological. In addition to supporting the need for occipitocervical fusion, these radiographic findings support the theory that the release of the posterior tension band causes progressive craniocervical settling, kyphosis, and progression of basilar invagination. Although our 2 patients presented with some ventral brainstem symptoms, they did not have clear signs of basilar invagination, and were in lower risk category groups as defined by Brockmeyer's treatment algorithm.

The radiographic markers discussed above assess macroinstability at the cervical vertebral junction. Although there is limited information available on macroinstability and its detection, resolution of symptoms 

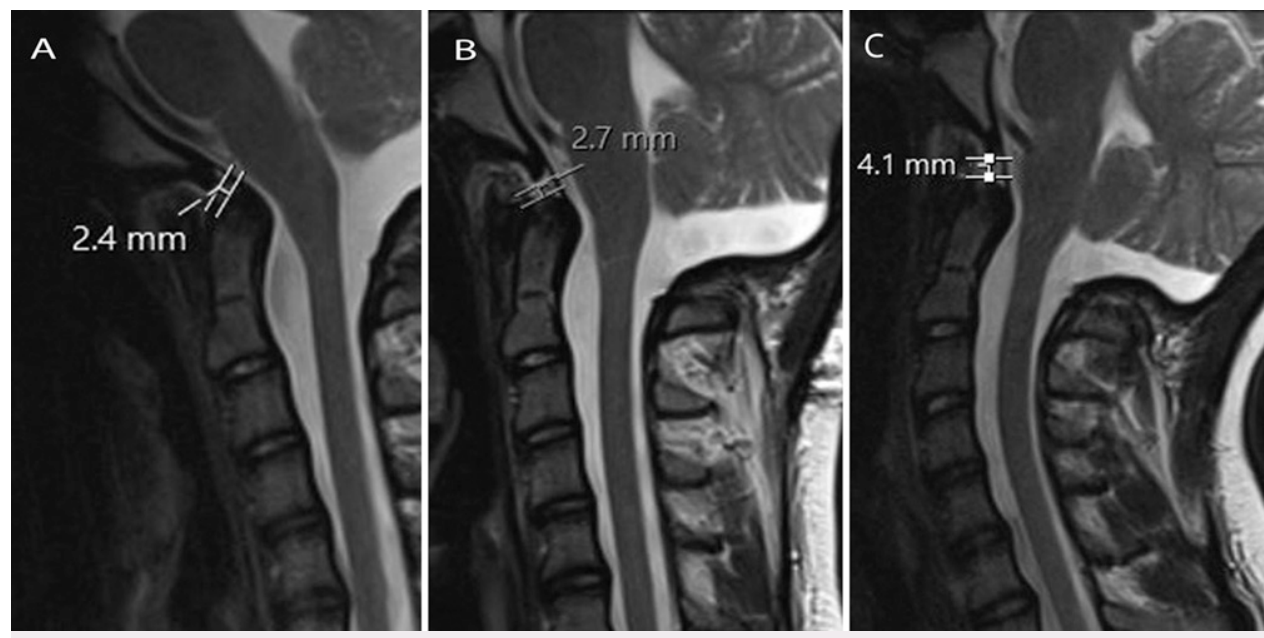

FIG. 5. Dynamic MRI with BDI measurements in flexion (A), neutral (B), and extension (C) positions.

with a C-collar argues that small micromotion in the setting of already abnormal anatomy can potentially cause irritation. A soft tissue pannus behind the dens is indicative of microinstability and may not be readily apparent on flexion-extension imaging. ${ }^{13}$ These 2 patients did not have evidence of macroinstability, so the theory of microinstability may explain the improvement in their symptoms with rigid C-collar immobilization.

As with all surgeries, occipitocervical fusions confer risk. The most commonly encountered perioperative complications are related to instrumentation failure, with nonunion rates found to be as high as $7 \%$ from occipitocervical fusion. ${ }^{14}$ Other encountered complications include injury to the vertebral artery, dural tears, and wound infection. A significant concern for many patients includes the restriction of movement at the craniocervical junction following fusion with a decrease in approximately $50 \%$ of all head and neck movements. ${ }^{15}$ Moreover, the long-term effects of posterior cervical spine fusion in the skeletally immature spine are not fully understood, and there are only a few published articles with long-term follow-up. ${ }^{16}$ Furthermore, occipitocervical fusions following a decompression are more complex surgeries than primary fusions. Following decompression, the occipital bone has been removed at midline, creating the need either to lateralize anchoring plates or to secure them higher. Lateralized plates will have to be placed in thinner bone than midline occipital bone, and securing the plates higher presents the problem of less muscular coverage. These considerations are not contraindications to fusions after decompression but rather add to a conservative approach to fusion Chiari patients after decompression. It should be noted that both patients in this article had extensive trials of nonoperative management of their symptoms without any improvement. They were extensively counseled on the lifelong implications of occipitocervical fusion prior to proceeding with any surgical intervention, including limitations in neck motion as well as the restriction of future activities.

\section{Lessons}

In our patients, improvement of symptoms with only rigid C-collar immobilization supported the decision to proceed with occipitocervical fusion. However, it is important to note that the failure of symptoms to improve following rigid C-collar immobilization would not suggest that an occipitocervical fusion would be unsuccessful. If no improvement is attained with C-collar immobilization, further evaluation of nonoperative evaluation strategies such as halo orthosis may be a consideration in these patients with complex Chiari malformations who do not have clear evidence of instability. Ultimately, we believe that a C-collar trial is a noninvasive, inexpensive evaluation and treatment approach that can provide assurance for the need of occipitocervical fusions in Chiari patients with ventral brainstem symptoms. This trial of a C-collar may be especially reassuring in similar patients without radiographic support of cervical vertebral junction instability. In addition, occipitocervical fusion, particularly in a pediatric population, conveys lifelong restrictions on neck rotation and flexion/extension as well as potential lifelong restrictions on activities. Any family presented with this option should be counseled extensively on the potential risks weighed against the known lifelong morbidity of this surgery.

\section{References}

1. Bonney PA, Maurer AJ, Cheema AA, et al. Clinical significance of changes in $\mathrm{pB}-\mathrm{C} 2$ distance in patients with Chiari type I malformations following posterior fossa decompression: a singleinstitution experience. J Neurosurg Pediatr. 2016;17(3):336-342.

2. Klekamp J. Neurological deterioration after foramen magnum decompression for Chiari malformation type I: old or new pathology? J Neurosurg Pediatr. 2012;10(6):538-547.

3. Grabb PA, Mapstone TB, Oakes WJ. Ventral brain stem compression in pediatric and young adult patients with Chiari I malformations. Neurosurgery. 1999;44(3):520-528.

4. Ridder T, Anderson RC, Hankinson TC. Ventral decompression in Chiari malformation, basilar invagination, and related disorders. Neurosurg Clin N Am. 2015;26(4):571-578.

5. Tubbs RS, Iskandar BJ, Bartolucci AA, et al. A critical analysis of the Chiari 1.5 malformation. J Neurosurg. 2004;101(2 suppl): 179-183.

6. Bollo RJ, Riva-Cambrin J, Brockmeyer MM, et al. Complex Chiari malformations in children: an analysis of preoperative risk factors for occipitocervical fusion. J Neurosurg Pediatr. 2012;10(2): 134-141.

7. Brockmeyer DL, Spader HS. Complex Chiari malformations in children: diagnosis and management. Neurosurg Clin N Am. 2015; 26(4):555-560. 
8. Ho WSC, Brockmeyer DL. Complex Chiari malformation: using craniovertebral junction metrics to guide treatment. Childs Nerv Syst. 2019;35(10):1847-1851.

9. Tubbs RS, Beckman J, Naftel RP, et al. Institutional experience with 500 cases of surgically treated pediatric Chiari malformation type I. J Neurosurg Pediatr. 2011;7(3):248-256.

10. Goldstein HE, Anderson RC. Craniovertebral junction instability in the setting of Chiari I malformation. Neurosurg Clin N Am. 2015; 26(4):561-569.

11. Menezes AH. Craniovertebral junction abnormalities with hindbrain herniation and syringomyelia: regression of syringomyelia after removal of ventral craniovertebral junction compression. J Neurosurg. 2012;116(2):301-309.

12. Smoker WR. Craniovertebral junction: normal anatomy, craniometry, and congenital anomalies. Radiographics. 1994;14(2): 255-277.

13. McClugage SG, Oakes WJ. The Chiari I malformation. J Neurosurg Pediatr. 2019;24(3):217-226.

14. Lall R, Patel NJ, Resnick DK. A review of complications associated with craniocervical fusion surgery. Neurosurgery. 2010; 67(5):1396-1403.
15. Lu DC, Roeser AC, Mummaneni VP, et al. Nuances of occipitocervical fixation. Neurosurgery. 2010;66(3 suppl):141-146.

16. Tauchi R, Imagama S, Ito Z, et al. Complications and outcomes of posterior fusion in children with atlantoaxial instability. Eur Spine J. 2012;21(7):1346-1352.

\section{Disclosures}

The authors report no conflict of interest concerning the materials or methods used in this study or the findings specified in this paper.

\section{Author Contributions}

Conception and design: Tomei, Elder. Acquisition of data: Tomei, Elder. Analysis and interpretation of data: all authors. Drafting the article: all authors. Critically revising the article: Tomei, Sunshine. Reviewed submitted version of manuscript: Tomei, Sunshine. Approved the final version of the manuscript on behalf of all authors: Tomei. Study supervision: Tomei.

\section{Correspondence}

Krystal L. Tomei: University Hospitals Rainbow Babies \& Children's Hospital, Cleveland, OH. krystal.tomei@uhhospitals.org. 